\title{
The Fourier coefficients of modular forms and Niebur modular integrals having small positive weight, I
}

\author{
by \\ Wladimir De Azevedo PriBitkin (Hackettstown, NJ)
}

Dedicated to the memory of my greatest teachers, my parents, Thereza de Azevedo Pribitkin and Edmund Pribitkin

1. Historical introduction. In 1989 Knopp [6] found explicit formulas for the Fourier coefficients of an arbitrary cusp form and more generally, but conditionally, of a holomorphic modular form (with a possible pole at $i \infty$ ) on the full modular group, $\Gamma(1)$, of weight $k, 4 / 3<k<2$, and multiplier system $v$. He assumed that there are no nontrivial cusp forms on $\Gamma(1)$ of complementary weight $2-k$ and conjugate multiplier system $\bar{v}$. In our initial paper we remove this assumption and capture the Fourier coefficients of an arbitrary "Niebur modular integral" on $\Gamma(1)$ of weight $k, 1<k<2$. En route we also obtain expressions for the Fourier coefficients of an arbitrary cusp form on $\Gamma(1)$ of weight $k, 0<k<1$. In particular we present formulas for the Fourier coefficients of $\eta^{r}(\tau), 0<r<2$, where $\eta(\tau)$ is the Dedekind eta-function.

An actual formula for the Fourier coefficients of an arbitrary modular form, even in the case of the full modular group, is not always available. For forms of weight greater than two the problem was solved by Petersson [11], who introduced the (parabolic) Poincaré series. Additionally, by considering a nonanalytic version of this series, he derived the coefficients of certain forms of weight two [12]. By integrating one of these forms, Petersson [12, p. 202] was the first to find the coefficients of the absolute modular invariant $J(\tau)$. For forms of negative weight Rademacher and Zuckerman [18] discovered expressions for the coefficients by relying on the circle method. Furthermore, Rademacher [15] employed a sharpened version of this method to rediscover Petersson's formula for $J(\tau)$. We remark that both approaches

1991 Mathematics Subject Classification: Primary 11F30. 
depend on nontrivial growth estimates for Kloosterman sums. Indeed, in all of the cases mentioned above, the Fourier coefficients are given by infinite series containing generalized Kloosterman sums.

Rademacher also established the modular invariance of $J(\tau)$ directly from its Fourier expansion [16, 17]. Later Knopp [3, 4] showed that although every modular form on $\Gamma(1)$ of nonpositive integral weight must have a Fourier series of a known type, the converse is false. However, these expansions are always Eichler integrals. In 1968 Niebur extended Knopp's work to include all negative real weights $[9,10]$. He also generalized the definition of modular forms (and certain Eichler integrals) to include his "new" modular integrals. Niebur's work revealed, in a rather explicit way, the duality which exists between Niebur modular integrals of negative weight and cusp forms of weight greater than two.

In this and subsequent work we obtain the Fourier coefficients of all Niebur modular integrals (including forms) on $\Gamma(1)$ having positive weight less than two. Not surprisingly, these coefficients have the "expected" representation involving Bessel functions and generalized Kloosterman sums. In this paper, we begin by considering the nonanalytic Poincaré series mentioned earlier and apply the Poisson summation formula to transform it into a Fourier series. This resulting expansion contains Selberg's Kloosterman zeta-function, which was studied in the 60's by Selberg [20]. It was not until the early 80's, however, that Goldfeld and Sarnak jointly established excellent results on the order of growth of this function [1]. By using these results we construct an analytic continuation of our Fourier series. We next evaluate this continuation at an appropriate point, and then by considering only the analytic "piece", we calculate the desired coefficients. We prove a decomposition theorem for all Niebur modular integrals on $\Gamma(1)$ having weight $k, 1<k<2$. We also display a duality between Niebur modular integrals of weight $k$ and cusp forms of weight $2-k$. We conclude this paper with some results which exploit known facts concerning cusp forms of small weight on $\Gamma(1)$. In a sequel we shall present analogous work on Niebur modular integrals on $\Gamma(1)$ of weight $k, 0<k \leq 1$.

2. Preliminary definitions and notation. The full modular group, $\Gamma(1)$, will refer to both the matrix group $\mathrm{SL}(2, \mathbb{Z})$ and the transformation group $\operatorname{PSL}(2, \mathbb{Z}) \simeq \mathrm{SL}(2, \mathbb{Z}) /\{ \pm I\}$. This should cause no confusion. Note that $\Gamma(1)$ is generated by $S=\left(\begin{array}{ll}1 & 1 \\ 0 & 1\end{array}\right)$ and $T=\left(\begin{array}{cc}0 & -1 \\ 1 & 0\end{array}\right)$. We define the real power of a nonzero complex number by $z^{r}=e^{r(\log |z|+i \arg z)}$, where $-\pi \leq \arg z<\pi$ and $\log |z|$ is real. Throughout, $k$ is a real number and $v$ is a multiplier system $(M S)$ for $\Gamma(1)$ in the weight $k$. Specifically, $v$ is a function from the matrix group $\Gamma(1)$ into the unit circle which, for $\tau \in \mathcal{H}$, the upper half-plane, satisfies the "consistency condition" 


$$
v\left(M_{1} M_{2}\right)\left(c_{3} \tau+d_{3}\right)^{k}=v\left(M_{1}\right) v\left(M_{2}\right)\left(c_{1} M_{2} \tau+d_{1}\right)^{k}\left(c_{2} \tau+d_{2}\right)^{k},
$$

where $M_{j}=\left(\begin{array}{cc}* & * \\ c_{j} & d_{j}\end{array}\right) \in \Gamma(1)$, for $j=1,2$, and $M_{1} M_{2}=\left(\begin{array}{cc}* & * \\ c_{3} & d_{3}\end{array}\right)$. Connected with this MS is a parameter $\kappa$, which is determined from $v$ by

$$
v(S)=e^{2 \pi i \kappa}, \quad 0 \leq \kappa<1 .
$$

Note that the conjugate function $\bar{v}$ is a MS for $\Gamma(1)$ in the weight $2-k$ and also that $\kappa=0$ can occur only when $k$ is an even integer.

Let $F$ be a function which is holomorphic in $\mathcal{H}$ and satisfies the modular relation

$$
F(V \tau)=v(V)(\gamma \tau+\delta)^{k} F(\tau), \quad \tau \in \mathcal{H}
$$

for all $V=\left(\begin{array}{ll}* & * \\ \gamma & \delta\end{array}\right) \in \Gamma(1)$. If $F$ also has a Fourier expansion of the type

$$
F(\tau)=\sum_{n=-\mu}^{\infty} a_{n} e^{2 \pi i(n+\kappa) \tau} \quad \forall \tau \in \mathcal{H}
$$

then $F$ is meromorphic at $\infty$ and we call $F$ a modular form on $\Gamma(1)$ of weight $k$ and $M S v$. If $F$ is not identically zero, then we assume that $a_{-\mu} \neq 0$ and we say that the order at $\infty$ of $F$ is $-\mu+\kappa$. The vector space of modular forms on $\Gamma(1)$ of weight $k$ and MS $v$ which have order at $\infty$ greater than or equal to $-\mu+\kappa$ is denoted by $C(\mu, k, v)$, and the space of cusp forms, $C(-\lfloor 1-\kappa\rfloor, k, v)$, is denoted by $C^{0}(k, v)$. Here $\lfloor\cdot\rfloor$ is the greatest integer function.

A Niebur modular integral on $\Gamma(1)$ of weight $k$ and $M S v$ is a function $F$ which is holomorphic in $\mathcal{H}$, meromorphic at $\infty$, and for which there exists a $G \in C^{0}(2-k, \bar{v})$ such that

$$
\left[F(\tau)-\bar{v}(V)(\gamma \tau+\delta)^{-k} F(V \tau)\right]^{-}=\int_{V^{-1}(\infty)}^{i \infty} G(z)(z-\bar{\tau})^{-k} d z
$$

for all $V \in \Gamma(1)$ and $\tau \in \mathcal{H}$. Here $[\cdot]^{-}$denotes complex conjugation and the path of integration is a vertical line in $\mathcal{H}$. (If $V$ is a translation, then the right-hand side is defined to be identically zero.) The vector space of Niebur modular integrals on $\Gamma(1)$ of weight $k$ and MS $v$ which have order at $\infty$ greater than or equal to $-\mu+\kappa$ is denoted by $I(\mu, k, v)$. Clearly, $C(\mu, k, v)$ is a subspace of $I(\mu, k, v)$. Since there are no nontrivial cusp forms of nonpositive weight, we have $I(\mu, k, v)=C(\mu, k, v)$ for $k \geq 2$.

The above type of modular integral was initially studied (for negative weights) by Douglas Niebur $[9,10]$, who showed that $F$ is a modular form if and only if $G$ is identically zero. This result holds for nonnegative weights as well and implies that each Niebur modular integral has a unique cusp form corresponding to it. 
3. Nonanalytic Poincaré series. We now define the functions which are at the heart of our investigation. Let $k \in \mathbb{R}$ and $\nu \in \mathbb{Z}$. For $\tau \in \mathcal{H}$ and $\operatorname{Re}(s)>2-k$ put

$$
P_{\nu}(\tau \mid s)=P_{\nu}(\tau \mid s ; k, v)=\sum_{\substack{c, d \in \mathbb{Z} \\(c, d)=1}} \frac{e^{2 \pi i(\nu+\kappa) M \tau}}{v(M)(c \tau+d)^{k}|c \tau+d|^{s}},
$$

where $M=\left(\begin{array}{ll}* & * \\ c & d\end{array}\right) \in \Gamma(1)$. The functions $P_{\nu}$ are called nonanalytic Poincaré series. Although $P_{\nu}(\tau \mid s)$ is not holomorphic in $\tau$, it is holomorphic in the complex variable $s$ for $\operatorname{Re}(s)>2-k$. Furthermore, $P_{\nu}(\tau \mid s)$ satisfies the transformation relation

(2) $P_{\nu}(V \tau \mid s)=v(V)(\gamma \tau+\delta)^{k}|\gamma \tau+\delta|^{s} P_{\nu}(\tau \mid s), \quad \tau \in \mathcal{H}, \operatorname{Re}(s)>2-k$,

for all $V=\left(\begin{array}{ll}* & * \\ \gamma & \delta\end{array}\right) \in \Gamma(1)$.

4. Selberg's Kloosterman zeta-function. Let $k \in \mathbb{R}$ and $m, n \in \mathbb{Z}$. For $\operatorname{Re}(w)>1$ put

$$
Z_{m, n}(w)=Z(w ; m, n, k, v)=\sum_{c=1}^{\infty} \frac{A_{c}(m, n)}{c^{2 w}},
$$

where

$$
A_{c}(m, n)=A(c ; m, n, k, v)=\sum_{\substack{-d=0 \\(c, d)=1}}^{c-1} \bar{v}(M) e^{\frac{2 \pi i}{c}[(m+\kappa) a+(n+\kappa) d]}
$$

is the generalized Kloosterman sum and $M=\left(\begin{array}{ll}a & * \\ c & d\end{array}\right) \in \Gamma(1)$. The Dirichlet series $Z_{m, n}(w)$ is called Selberg's Kloosterman zeta-function and is inextricably linked to nonanalytic Poincaré series. The properties of $Z_{m, n}(w)$ are summarized in this section. In his seminal work Selberg [20] obtained

Lemma 1. Let $Z_{m, n}(w)$ be defined by (3) and (4).

(a) The sum defining $Z_{m, n}(w)$ converges absolutely and uniformly on compacta in $\operatorname{Re}(w)>1$ and so $Z_{m, n}(w)$ is holomorphic in $\operatorname{Re}(w)>1$.

(b) $Z_{m, n}(w)$ has an analytic continuation to a function meromorphic in the whole w-plane.

(c) If $m+\kappa \neq 0$ and $n+\kappa \neq 0$, then $Z_{m, n}(w)$ is holomorphic in $\operatorname{Re}(w)>$ $1 / 2$ with the possible exception of a finite set of simple poles on the real segment $1 / 2<w<1$ (the "exceptional poles").

The exposition in [20], though beautiful, is skeletal and assumes that $k$, $m+\kappa$ and $n+\kappa$ are positive. A fleshed out proof of the above lemma can 
be found in [14]. Of particular importance is the simple observation that

$$
\bar{Z}(\bar{w} ; m, n, k, v)= \begin{cases}Z(w ;-m-1,-n-1,-k, \bar{v}) & \text { if } 0<\kappa<1, \\ Z(w ;-m,-n,-k, \bar{v}) & \text { if } \kappa=0 .\end{cases}
$$

The following derives from Lemma 1 and Roelcke's result [19] that the spectrum of the Casimir operator $\Delta_{k}=y^{2}\left(\partial^{2} / \partial x^{2}+\partial^{2} / \partial y^{2}\right)-i k y \partial / \partial x$ is contained in the half-line $[(|k| / 2)(1-|k| / 2), \infty)$.

Lemma 2. Let $0<k<2$. Then for all integers $m$ and $n, Z(w ; m, n, k, v)$ is holomorphic in $\operatorname{Re}(w)>1 / 2+|k-1| / 2$. Moreover, we have the following:

(a) Let $0<k<1$. For all integers $m$ and $n, Z(w ; m, n, k, v)$ is holomorphic in $\operatorname{Re}(w) \geq 1-k / 2$ with the possible exception of a simple pole at $1-k / 2$. This pole occurs if and only if $m$ and $n$ are both nonnegative and there exists $f \in C^{0}(k, v)$ such that the terms $e^{2 \pi i(m+\kappa) \tau}$ and $e^{2 \pi i(n+\kappa) \tau}$ both occur in its Fourier expansion.

(b) Let $k=1$. For all integers $m$ and $n, Z(w ; m, n, 1, v)$ is holomorphic in $\operatorname{Re}(w)>1 / 2$ and at $w=1 / 2$ has at most a simple pole. This pole occurs if and only if either $m$ and $n$ are both nonnegative and there exists $f \in C^{0}(1, v)$ such that the terms $e^{2 \pi i(m+\kappa) \tau}$ and $e^{2 \pi i(n+\kappa) \tau}$ both occur in its Fourier expansion or $m$ and $n$ are both negative and there exists $f \in C^{0}(1, \bar{v})$ such that the terms $e^{2 \pi i[(-m-1)+(1-\kappa)] \tau}$ and $e^{2 \pi i[(-n-1)+(1-\kappa)] \tau}$ both occur in its Fourier expansion.

(c) Let $1<k<2$. For all integers $m$ and $n, Z(w ; m, n, k, v)$ is holomorphic in $\operatorname{Re}(w) \geq k / 2$ with the possible exception of a simple pole at $k / 2$. This pole occurs if and only if $m$ and $n$ are both negative and there exists $f \in$ $C^{0}(2-k, \bar{v})$ such that the terms $e^{2 \pi i[(-m-1)+(1-\kappa)] \tau}$ and $e^{2 \pi i[(-n-1)+(1-\kappa)] \tau}$ both occur in its Fourier expansion.

Goldfeld and Sarnak [1] established the important

Lemma 3. Let $k \in \mathbb{R}$ and $m, n \in \mathbb{Z}$.

(a) If $\operatorname{Re}(w) \geq 1+\delta, \delta>0$, then $\left|Z_{m, n}(w)\right| \leq \zeta(1+2 \delta)$. In particular, if $\operatorname{Re}(w) \geq 3 / 2$, then $\left|Z_{m, n}(w)\right| \leq \pi^{2} / 6$.

(b) Assume now that $m+\kappa \neq 0$ and $n+\kappa \neq 0$. If $1 / 2<\operatorname{Re}(w)<3 / 2$ and $|\operatorname{Im}(w)| \geq 1$, then

$$
\left|Z_{m, n}(w)\right| \leq C \frac{|m+\kappa| \cdot|n+\kappa| \cdot|\operatorname{Im}(w)|^{1 / 2}}{\operatorname{Re}(w)-1 / 2},
$$

where $C$ is a positive constant depending at most upon $k$ and $\kappa$.

By using the above bound on $\left|Z_{m, n}(w)\right|$ Knopp $[5,6]$ proved 
Lemma 4. Let $0<k<2$ and $m, n \in \mathbb{Z}$.

(a) If $0<k<2 / 3$ and $Z_{m, n}(w)$ is holomorphic at $w=1-k / 2$, then

$$
Z_{m, n}(1-k / 2)=\sum_{c=1}^{\infty} \frac{A_{c}(m, n)}{c^{2-k}}
$$

(b) If $4 / 3<k<2$ and $Z_{m, n}(w)$ is holomorphic at $w=k / 2$, then

$$
Z_{m, n}(k / 2)=\sum_{c=1}^{\infty} \frac{A_{c}(m, n)}{c^{k}}
$$

Because $Z(w ; m, n, k, v)=Z(w ; m, n, k+2 l, v)$ for all $l \in \mathbb{Z}$, both Lemma 2 and Lemma 4 may be generalized to include other noneven real weights.

As an aside we comment on $Z_{m, n}(w)$ for the case where $m+\kappa=0$ or $n+\kappa=0$. Assume that $m+\kappa=0$. This implies that $k$ is an even integer (say, zero) and $v$ is identically 1 and so the generalized Kloosterman sum reduces to either Euler's totient (if $n=0$ ) or Ramanujan's sum (if $n \neq 0$ ). From this it follows that

$$
Z(w ; n)=Z(w ; 0, n, 0,1)= \begin{cases}\frac{\zeta(2 w-1)}{\zeta(2 w)} & \text { if } n=0 \\ \frac{\sigma_{1-2 w}(n)}{\zeta(2 w)} & \text { if } n \neq 0\end{cases}
$$

for $\operatorname{Re}(w)>1$, where $\sigma_{1-2 w}(n)=\sum_{d \mid n, d>0} d^{1-2 w}$ is the divisor function. Well-known facts and speculations concerning the Riemann zeta-function can be used to study $Z(w ; n)$. Furthermore, if $n+\kappa=0$, then the above carries over, because $Z(w ; m, 0,0,1)=Z(w ; 0, m, 0,1)$.

5. Fourier expansion. The connection between Selberg's Kloosterman zeta-function and nonanalytic Poincaré series will now be made explicit. From (2) it follows that $P_{\nu}(\tau \mid s)$ has a Fourier expansion of the type

$$
P_{\nu}(\tau \mid s)=\sum_{n=-\infty}^{\infty} a_{n}(y, s) e^{2 \pi i(n+\kappa) x}, \quad \text { where } \tau=x+y i
$$

Lemma 5. Let $P_{\nu}$ be defined by (1) and assume that $\kappa \neq 0$. Then for $\operatorname{Re}(s)>2-k$ we have

$$
\begin{aligned}
P_{\nu}(\tau \mid s)= & 2 e^{2 \pi i(\nu+\kappa) \tau}+2 i^{-k} \frac{(2 \pi)^{k+s}}{\Gamma(s / 2)} \\
& \times\left\{\sum_{n=0}^{\infty}(n+\kappa)^{k+s-1} e^{2 \pi i(n+\kappa) \tau} \sum_{p=0}^{\infty} \frac{\left[-4 \pi^{2}(n+\kappa)(\nu+\kappa)\right]^{p}}{p ! \Gamma(k+p+s / 2)}\right. \\
& \times \sigma(4 \pi(n+\kappa) y, k+p+s / 2, s / 2) Z_{\nu, n}(s / 2+k / 2+p)
\end{aligned}
$$




$$
\begin{aligned}
& +\sum_{n=1}^{\infty}(n-\kappa)^{k+s-1} e^{-2 \pi i(n-\kappa) \bar{\tau}} \sum_{p=0}^{\infty} \frac{\left[-4 \pi^{2}(n-\kappa)(\nu+\kappa)\right]^{p}}{p ! \Gamma(k+p+s / 2)} \\
& \left.\times \sigma(4 \pi(n-\kappa) y, s / 2, k+p+s / 2) Z_{\nu,-n}(s / 2+k / 2+p)\right\} .
\end{aligned}
$$

Here,

$$
\sigma(\eta, \alpha, \beta)=\int_{0}^{\infty}(u+1)^{\alpha-1} u^{\beta-1} e^{-\eta u} d u
$$

and $Z_{\nu, \pm n}$ is Selberg's Kloosterman zeta-function.

The function $\sigma(\eta, \alpha, \beta)$ is holomorphic in $\operatorname{Re}(\eta)>0$, entire in $\alpha$ and holomorphic in $\operatorname{Re}(\beta)>0$. Its well-known relative, the (exponentially decaying) Whittaker function,

$$
W_{(\alpha-\beta) / 2,(\alpha+\beta-1) / 2}(\eta)=\eta^{(\alpha+\beta) / 2} e^{-\eta / 2} \frac{\sigma(\eta, \alpha, \beta)}{\Gamma(\beta)},
$$

is entire in $\alpha$ and in $\beta$. The preceding lemma, which was established by Knopp [6], is largely an application of the Poisson summation formula. In particular, the following integral formula (see [2, p. 367] and use (7)) is needed:

$$
\begin{aligned}
& \int_{-\infty}^{\infty} \frac{e^{-2 \pi i \lambda u}}{(u+y i)^{\alpha}(u-y i)^{\beta}} d u \\
& = \begin{cases}\frac{2 \pi(-i)^{\alpha-\beta} \Gamma(\alpha+\beta-1)}{\Gamma(\alpha) \Gamma(\beta)}(2 y)^{1-\alpha-\beta} & \text { if } \lambda=0, \\
\frac{(2 \pi)^{\alpha+\beta}(-i)^{\alpha-\beta} \lambda^{\alpha+\beta-1}}{\Gamma(\alpha) \Gamma(\beta)} e^{-2 \pi \lambda y} \sigma(4 \pi \lambda y, \alpha, \beta) & \text { if } \lambda>0, \\
\frac{(2 \pi)^{\alpha+\beta}(-i)^{\alpha-\beta}(-\lambda)^{\alpha+\beta-1}}{\Gamma(\alpha) \Gamma(\beta)} e^{2 \pi \lambda y} \sigma(-4 \pi \lambda y, \beta, \alpha) & \text { if } \lambda<0 .\end{cases}
\end{aligned}
$$

Here $\lambda \in \mathbb{R}, \operatorname{Re}(\alpha+\beta)>1$ and $y>0$. The analog of Lemma 5 for $\kappa=0$ can be derived by using (8). Because we are interested in the range $0<k<2$ we shall not need this case.

We conclude this section by recording the following simple result, which can be proved by using the definition of the gamma function.

Lemma 6. Let $\sigma(\eta, \alpha, \beta)$ be defined by (6) and assume that $\operatorname{Re}(\eta)>0$ and that $\operatorname{Re}(\beta)>0$.

(a) If $\operatorname{Re}(\alpha) \leq 1$, then

$$
|\sigma(\eta, \alpha, \beta)| \leq \frac{\Gamma(\operatorname{Re}(\beta))}{[\operatorname{Re}(\eta)]^{\operatorname{Re}(\beta)}} .
$$


(b) If $\operatorname{Re}(\alpha)>1$, then

$$
|\sigma(\eta, \alpha, \beta)|<2^{\operatorname{Re}(\alpha)-1}\left\{\frac{1}{\operatorname{Re}(\beta)}+\frac{\Gamma(\operatorname{Re}(\alpha+\beta)-1)}{[\operatorname{Re}(\eta)]^{\operatorname{Re}(\alpha+\beta)-1}}\right\} .
$$

\section{Analytic continuation}

Lemma 7. Let $P_{\nu}(\tau \mid s)$ be defined by (1), where $\nu \in \mathbb{Z}$, and assume that $1<k<2$. Then $P_{\nu}(\tau \mid s)$ has an analytic continuation in $s$ into the closed half-plane $\operatorname{Re}(s) \geq 0$.

Proof. Our proof generalizes the one given by Knopp [6] for $\nu \geq 0$ and $4 / 3<k<2$. We shall carry out an analytic continuation of the Fourier expansion (Lemma 5) of $P_{\nu}(\tau \mid s)$ into an open half-plane containing $s=0$. We first observe that the factors preceding both infinite sums on $n$ are entire functions of $s$ and, in fact, have a simple zero at $s=0$ (arising from $1 / \Gamma(s / 2)$ ). Secondly, we note that all of the factors in both summands are entire functions of $s$ except for the " $\sigma$-functions" and the zeta-functions. Because $\sigma(\eta, \alpha, \beta) / \Gamma(\beta)$ is an entire function in $\alpha$ and in $\beta$, however, the $\sigma$-functions do not pose any serious difficulties. The function $\sigma(4 \pi(n-\kappa) y, s / 2, k+p+s / 2)$ is analytic in $\operatorname{Re}(s) \geq-2$ (since $p \geq 0$ and $k>1$ ), and the function

$$
\frac{\sigma(4 \pi(n+\kappa) y, k+p+s / 2, s / 2)}{\Gamma(s / 2)}
$$

has an analytic continuation to $\operatorname{Re}(s)>-2$ explicitly given by

$$
\begin{aligned}
& \frac{1}{\Gamma(s / 2+1)}[4 \pi(n+\kappa) y \cdot \sigma(4 \pi(n+\kappa) y, k+p+s / 2, s / 2+1) \\
& \quad-(k+p+s / 2-1) \cdot \sigma(4 \pi(n+\kappa) y, k+p+s / 2-1, s / 2+1)] .
\end{aligned}
$$

We next examine the zeta-functions by invoking Lemma 2 (c). If $\nu \geq 0$, then both $Z_{\nu, n}(s / 2+k / 2+p)$ and $Z_{\nu,-n}(s / 2+k / 2+p)$ are holomorphic in $\operatorname{Re}(s) \geq 0$ (because $p \geq 0$ ). If $\nu<0$, then $Z_{\nu, n}(s / 2+k / 2+p)$ is holomorphic in $\operatorname{Re}(s) \geq 0$, but $Z_{\nu,-n}(s / 2+k / 2+p)$ is holomorphic in $\operatorname{Re}(s) \geq 0$ with the possible exception of a simple pole at $s=0$ (which is a concern only for $p=0)$. We note, however, that $Z_{\nu,-n}(s / 2+k / 2) / \Gamma(s / 2)$ is always analytic at $s=0$. Since the locations of the potential poles (in $\operatorname{Re}(s)<0$ ) of both $Z_{\nu, n}$ and $Z_{\nu,-n}$ are independent of $n$ (see [20]) and since there are at most finitely many potential poles in $1-k<\operatorname{Re}(s)<0$ (see Lemma $1(\mathrm{c})$ ), there exists $\delta^{*}>0$ (independent of $n$ ) such that both $Z_{\nu, n}(s / 2+k / 2+p)$ and $[\Gamma(s / 2)]^{-1} Z_{\nu,-n}(s / 2+k / 2+p)$ are holomorphic in $\operatorname{Re}(s)>-\delta^{*}$. We now set $\delta=\min \left(\delta^{*}, k-1\right)$ and observe that, once we pull in the function $1 / \Gamma(s / 2)$, both major summands on the right-hand side of the Fourier expansion of $P_{\nu}(\tau \mid s)$ are analytic in $\operatorname{Re}(s)>-\delta$. 
In order to conclude the proof, it suffices to show that, once $1 / \Gamma(s / 2)$ is pulled in and (9) is used, both infinite sums on $n$ converge uniformly in compact subsets of $-\delta<\operatorname{Re}(s)<1$. But this can be established by using Lemma 3, Lemma 6 and basic properties of the gamma function.

Lemma 8. Let $P_{\nu}(\tau)=P_{\nu}(\tau \mid 0)$. Then for $\nu \in \mathbb{Z}$ and $1<k<2$ we have $P_{\nu}(\tau)=G_{\nu}(\tau)+R_{\nu}(\tau)$, where

$$
G_{\nu}(\tau)=G_{\nu}(\tau ; k, v)=2 e^{2 \pi i(\nu+\kappa) \tau}+\sum_{n=0}^{\infty} a_{n}(\nu, k, v) e^{2 \pi i(n+\kappa) \tau}
$$

with

$$
a_{n}(\nu, k, v)=2 i^{-k}(2 \pi)^{k}(n+\kappa)^{k-1} \sum_{p=0}^{\infty} \frac{\left[-4 \pi^{2}(n+\kappa)(\nu+\kappa)\right]^{p}}{p ! \Gamma(k+p)} Z_{\nu, n}(k / 2+p)
$$

and

with

$$
R_{\nu}(\tau)=R_{\nu}(\tau ; k, v)=\sum_{n=1}^{\infty} b_{n}(y ; \nu, k, v) e^{-2 \pi i(n-\kappa) \tau}
$$

$$
b_{n}(y ; \nu, k, v)=-4 \pi i(2 y i)^{1-k} \operatorname{Res}\left(Z_{\nu,-n}(w) ; k / 2\right) \int_{1}^{\infty} u^{-k} e^{-4 \pi(n-\kappa) y u} d u .
$$

Proof. From (9) and integration by parts it follows that

$$
\left.\frac{\sigma(4 \pi(n+\kappa) y, k+p+s / 2, s / 2)}{\Gamma(s / 2)}\right|_{s=0}=1 .
$$

From the proof of Lemma 7 we find that

$$
\left.\frac{Z_{\nu,-n}(s / 2+k / 2+p)}{\Gamma(s / 2)}\right|_{s=0}
$$

equals zero for all $p$ except possibly $p=0$. Using these facts and Lemma 5 gives

$$
\begin{aligned}
P_{\nu}(\tau)= & G_{\nu}(\tau)+2 i^{-k}(2 \pi)^{k} \sum_{n=1}^{\infty}\left\{\frac{(n-\kappa)^{k-1}}{\Gamma(k)} \int_{0}^{\infty}(u+1)^{-1} u^{k-1} e^{-4 \pi(n-\kappa) y u} d u\right. \\
& \left.\times\left.\left[\frac{1}{\Gamma(s / 2)} Z_{\nu,-n}(s / 2+k / 2)\right]\right|_{s=0}\right\} e^{-2 \pi i(n-\kappa) \bar{\tau}} .
\end{aligned}
$$

Now, the proof follows from the observation that

$$
\int_{0}^{\infty}(u+1)^{-1} u^{k-1} e^{-4 \pi(n-\kappa) y u} d u=\frac{\Gamma(k) e^{4 \pi(n-\kappa) y}}{[4 \pi(n-\kappa) y]^{k-1}} \int_{1}^{\infty} w^{-k} e^{-4 \pi(n-\kappa) y w} d w .
$$

This can be shown by letting $(u+1)^{-1}=\int_{0}^{\infty} e^{-(u+1) t} d t$, interchanging integrals and setting $t=4 \pi(n-\kappa) y w-4 \pi(n-\kappa) y$. 
7. Cusp forms. For completeness we include the next theorem, which provides a slight generalization of the formulas found in [6].

Theorem 9. Let $G_{\nu}(\tau)$ be defined as in Lemma 8. Assume now that $\nu \geq 0$ and $1<k<2$. Then $G_{\nu}(\tau) \in C^{0}(k, v)$ and has the following Fourier expansion:

$$
G_{\nu}(\tau)=2 e^{2 \pi i(\nu+\kappa) \tau}+\sum_{n=0}^{\infty} a_{n}(\nu, k, v) e^{2 \pi i(n+\kappa) \tau}, \quad \tau \in \mathcal{H},
$$

where

$$
\begin{aligned}
a_{n}(\nu, k, v) & \\
= & 4 \pi i^{-k}\left\{\sum _ { c = 1 } ^ { \infty } \left[\frac{A_{c}(\nu, n)}{c}\left(\frac{n+\kappa}{\nu+\kappa}\right)^{(k-1) / 2} J_{k-1}\left(\frac{4 \pi}{c} \sqrt{(\nu+\kappa)(n+\kappa)}\right)\right.\right. \\
& \left.\left.-\frac{[2 \pi(n+\kappa)]^{k-1}}{\Gamma(k)} \cdot \frac{A_{c}(\nu, n)}{c^{k}}\right]+\frac{[2 \pi(n+\kappa)]^{k-1}}{\Gamma(k)} \cdot Z_{\nu, n}(k / 2)\right\} .
\end{aligned}
$$

Here

$$
J_{k-1}(u)=\sum_{p=0}^{\infty} \frac{(-1)^{p}(u / 2)^{2 p+k-1}}{p ! \Gamma(p+k)}, \quad u>0,
$$

is the Bessel function of the first kind of order $k-1$.

Proof. Recall that $P_{\nu}(\tau)=G_{\nu}(\tau)+R_{\nu}(\tau)$ and observe that $P_{\nu}(\tau)$ transforms like a modular form of weight $k$. But for $\nu \geq 0$ we have $R_{\nu}(\tau) \equiv 0$ and so $G_{\nu}(\tau) \equiv P_{\nu}(\tau)$ is a cusp form on $\Gamma(1)$ of weight $k$ and MS $v$. The Fourier expansion results from Lemma 8 , the fact that

$$
Z_{\nu, n}(k / 2+p)=\sum_{c=1}^{\infty} \frac{A_{c}(\nu, n)}{c^{k+2 p}} \quad \text { for } p \geq 1,
$$

and the definition of $J_{k-1}$.

COROllary 10. Let $G_{\nu}(\tau)$ be defined as before but now assume that $\nu \geq 0$ and $4 / 3<k<2$. Then $G_{\nu}(\tau) \in C^{0}(k, v)$ and has the following Fourier expansion:

$$
G_{\nu}(\tau)=2 e^{2 \pi i(\nu+\kappa) \tau}+\sum_{n=0}^{\infty} c_{n}(\nu, k, v) e^{2 \pi i(n+\kappa) \tau}, \quad \tau \in \mathcal{H},
$$

where

$$
c_{n}(\nu, k, v)=4 \pi i^{-k}\left(\frac{n+\kappa}{\nu+\kappa}\right)^{(k-1) / 2} \sum_{c=1}^{\infty} \frac{A_{c}(\nu, n)}{c} J_{k-1}\left(\frac{4 \pi}{c} \sqrt{(\nu+\kappa)(n+\kappa)}\right) .
$$

Proof. This is an immediate consequence of Lemma 4(b) and Theorem 9. 


\section{Niebur modular integrals}

TheOrem 11. Let $G_{\nu}(\tau)$ be defined as in Lemma 8. Assume now that $\nu<0$ and $1<k<2$.

(a) $G_{\nu}(\tau) \in I(-\nu, k, v)$. Specifically, for all $V=\left(\begin{array}{ll}* & * \\ \gamma & \delta\end{array}\right) \in \Gamma(1)$ and $\tau \in \mathcal{H}$ we have

$$
\left[G_{\nu}(\tau)-\bar{v}(V)(\gamma \tau+\delta)^{-k} G_{\nu}(V \tau)\right]^{-}=\int_{V^{-1}(\infty)}^{i \infty} H_{-\nu-1}^{*}(z)(z-\bar{\tau})^{-k} d z
$$

where

$$
\begin{aligned}
H_{-\nu-1}^{*}(z)= & (-1)^{1-k} 4 \pi i \\
& \times \sum_{n=0}^{\infty} \operatorname{Res}(Z(w ;-\nu-1, n, 2-k, \bar{v}) ; k / 2) e^{2 \pi i[n+(1-\kappa)] z}
\end{aligned}
$$

is in $C^{0}(2-k, \bar{v})$.

(b) $G_{\nu}(\tau)$ has the following Fourier expansion:

$$
G_{\nu}(\tau)=2 e^{-2 \pi i|\nu+\kappa| \tau}+\sum_{n=0}^{\infty} a_{n}(\nu, k, v) e^{2 \pi i(n+\kappa) \tau}, \quad \tau \in \mathcal{H}
$$

where

$$
\begin{aligned}
& a_{n}(\nu, k, v) \\
& =4 \pi i^{-k}\left\{\sum _ { c = 1 } ^ { \infty } \left[\frac{A_{c}(\nu, n)}{c}\left(\frac{n+\kappa}{|\nu+\kappa|}\right)^{(k-1) / 2} I_{k-1}\left(\frac{4 \pi}{c} \sqrt{|\nu+\kappa|(n+\kappa)}\right)\right.\right. \\
& \left.\left.\quad-\frac{[2 \pi(n+\kappa)]^{k-1}}{\Gamma(k)} \cdot \frac{A_{c}(\nu, n)}{c^{k}}\right]+\frac{[2 \pi(n+\kappa)]^{k-1}}{\Gamma(k)} \cdot Z_{\nu, n}(k / 2)\right\} .
\end{aligned}
$$

Here

$$
I_{k-1}(u)=\sum_{p=0}^{\infty} \frac{(u / 2)^{2 p+k-1}}{p ! \Gamma(p+k)}, \quad u>0,
$$

is the modified Bessel function of the first kind of order $k-1$.

Proof. The Fourier expansion in part (b) is easily derived from Lemma 8.

We now show part (a). Let $P_{\nu}(\tau)$ and $R_{\nu}(\tau)$ be defined as in Lemma 8 , so that $G_{\nu}(\tau)=P_{\nu}(\tau)-R_{\nu}(\tau)$. Because $\nu<0, R_{\nu}(\tau)$ need not be identically zero. We know that $P_{\nu}(\tau)$ transforms like a modular form of weight $k$ (but is not necessarily analytic in $\tau$ ) and so we must study $R_{\nu}(\tau)$ more closely. 
Starting from Lemma 8 and interchanging sum and integral we get

$$
\begin{aligned}
R_{\nu}(\tau) & =-4 \pi i(2 y i)^{1-k} \int_{1}^{\infty} w^{-k} h^{*}(2 i y w-\tau) d w \\
& =-4 \pi i \int_{-\bar{\tau}}^{i \infty} h^{*}(z)(z+\tau)^{-k} d z .
\end{aligned}
$$

(Note that we set $z=2 i y w-\tau$ and used the fact that $z+\tau \in \mathcal{H}$.) Here

$$
h^{*}(z)=\sum_{n=0}^{\infty} \operatorname{Res}\left(Z_{\nu,-n-1}(w) ; k / 2\right) e^{2 \pi i[n+(1-\kappa)] z} .
$$

Next, we replace $z$ with $-\bar{z}$ and complex-conjugate both sides to obtain

$$
\overline{R_{\nu}(\tau)}=(-1)^{1-k} 4 \pi i \int_{\tau}^{i \infty} \overline{h^{*}(-\bar{z})}(z-\bar{\tau})^{-k} d z .
$$

From the definition of $H_{-\nu-1}^{*}(z)$ and (5) it follows that

$$
\overline{R_{\nu}(\tau)}=\int_{\tau}^{i \infty} H_{-\nu-1}^{*}(z)(z-\bar{\tau})^{-k} d z .
$$

Let $V=\left(\begin{array}{ll}* & * \\ \gamma & \delta\end{array}\right) \in \Gamma(1)$. We have

$$
\begin{aligned}
\overline{R_{\nu}(V \tau)} & =\int_{V \tau}^{i \infty} H_{-\nu-1}^{*}(z)(z-V \bar{\tau})^{-k} d z \\
& =(\gamma \bar{\tau}+\delta)^{k} \int_{\tau}^{-\delta / \gamma} H_{-\nu-1}^{*}(V u)(\gamma u+\delta)^{k-2}(u-\bar{\tau})^{-k} d u
\end{aligned}
$$

where we let $z=V u$ and then used the fact that

$$
(V u-V \bar{\tau})^{-k}=\frac{(u-\bar{\tau})^{-k}}{(\gamma u+\delta)^{-k}(\gamma \bar{\tau}+\delta)^{-k}} .
$$

(It is crucial here that $u$ and $\tau$ are both in $\mathcal{H}$.) We now claim that $H_{-\nu-1}^{*}(z)$ is a cusp form of weight $2-k$ and $\mathrm{MS} \bar{v}$ and so

$$
\overline{R_{\nu}(V \tau)}=\bar{v}(V)(\gamma \bar{\tau}+\delta)^{k} \int_{\tau}^{-\delta / \gamma} H_{-\nu-1}^{*}(u)(u-\bar{\tau})^{-k} d u
$$

The required modular relation for $G_{\nu}$ now follows from (10), (11) and an application of Cauchy's Theorem.

Our claim that $H_{-\nu-1}^{*}(z) \in C^{0}(2-k, \bar{v})$ is substantiated in the next section by Theorem 14. To see that $H_{-\nu-1}^{*}$ is a constant multiple of $H_{\nu}$, replace $\nu, k, v$, and $\kappa$ with $-\nu-1,2-k, \bar{v}$, and $1-\kappa$, respectively. 
Corollary 12. Let $G_{\nu}(\tau)$ be defined as before but now assume that $\nu<0$ and $4 / 3<k<2$. Then $G_{\nu}(\tau) \in I(-\nu, k, v)$ and has the following Fourier expansion:

$$
G_{\nu}(\tau)=2 e^{-2 \pi i|\nu+\kappa| \tau}+\sum_{n=0}^{\infty} c_{n}(\nu, k, v) e^{2 \pi i(n+\kappa) \tau}, \quad \tau \in \mathcal{H}
$$

where

$c_{n}(\nu, k, v)=4 \pi i^{-k}\left(\frac{n+\kappa}{|\nu+\kappa|}\right)^{(k-1) / 2} \sum_{c=1}^{\infty} \frac{A_{c}(\nu, n)}{c} I_{k-1}\left(\frac{4 \pi}{c} \sqrt{|\nu+\kappa|(n+\kappa)}\right)$.

Proof. This follows from Lemma 4(b), and Theorem 11(b).

9. Cusp forms revisited. In order to obtain cusp forms of weight between zero and one it is convenient to consider $Q_{\nu}(\tau \mid s)=Q_{\nu}(\tau \mid s ; k, v)=$ $y^{s / 2} P_{\nu}(\tau \mid s)$, where $P_{\nu}$ is defined by $(1)$ and $y=\operatorname{Im}(\tau)$. Observe that, for $\tau \in \mathcal{H}$ and $\operatorname{Re}(s)>2-k$, we have

$$
Q_{\nu}(\tau \mid s)=\sum_{\substack{c, d \in \mathbb{Z} \\(c, d)=1}} \frac{e^{2 \pi i(\nu+\kappa) M \tau}}{v(M)(c \tau+d)^{k}} \cdot[\operatorname{Im}(M \tau)]^{s / 2},
$$

where $M=\left(\begin{array}{ll}* & * \\ c & d\end{array}\right) \in \Gamma(1)$, and so

$$
Q_{\nu}(V \tau \mid s)=v(V)(\gamma \tau+\delta)^{k} Q_{\nu}(\tau \mid s), \quad \tau \in \mathcal{H}, \operatorname{Re}(s)>2-k,
$$

for all $V=\left(\begin{array}{ll}* & * \\ \gamma & \delta\end{array}\right) \in \Gamma(1)$. That is, $Q_{\nu}(\tau \mid s)$ transforms like a modular form (but is not holomorphic in $\tau$ ). The Fourier expansion for $Q_{\nu}(\tau \mid s)$ follows immediately from that of $P_{\nu}(\tau \mid s)$, which is given in Lemma 5 . Because we are now focusing on a different weight range, however, we shall need the following result concerning the analyticity of $Q_{\nu}(\tau \mid s)$ in $s$.

Lemma 13. Let $Q_{\nu}(\tau \mid s)$ be defined by (12). Assume that $\nu \in \mathbb{Z}, \nu \geq 0$, and $0<k<1$. Then $Q_{\nu}(\tau \mid s)$ has an analytic continuation in $s$ into the closed half-plane $\operatorname{Re}(s) \geq 2-2 k$, with the possible exception of a simple pole at $s=2-2 k$.

Pr o of. Our proof here is akin to the one already given for Lemma 7 and so it will be brief. We shall examine the Fourier expansion of $y^{-s / 2} Q_{\nu}(\tau \mid s)=$ $P_{\nu}(\tau \mid s)$ stated in Lemma 5. As before, the $\sigma$-functions are easy to handle. The functions $\sigma(4 \pi(n+\kappa) y, k+p+s / 2, s / 2)$ and $\sigma(4 \pi(n+\kappa) y, s / 2, k+p+s / 2)$ are analytic in $\operatorname{Re}(s)>0$ and $\operatorname{Re}(s) \geq 0$, respectively. Since $0<k<1$, both are clearly analytic in $\operatorname{Re}(s) \geq 2-2 k$. We now analyse the zeta-functions by recalling results from Section 4 . For $p \geq 1$ we trivially see that both $Z_{\nu, n}(s / 2+k / 2+p)$ and $Z_{\nu,-n}(s / 2+k / 2+p)$ are holomorphic in $\operatorname{Re}(s) \geq-k$. So the critical case occurs when $p=0$. By employing Lemma 2(a) and the 
fact that $\nu \geq 0$, we find that $Z_{\nu,-n}(s / 2+k / 2)$ is analytic for $\operatorname{Re}(s) \geq 2-2 k$ and that $Z_{\nu, n}(s / 2+k / 2)$ is analytic for $\operatorname{Re}(s) \geq 2-2 k$ with the possible exception of a simple pole at $s=2-2 k$. The above facts, coupled with the growth estimates from Lemmas 3 and 6 , can be used to finish the proof.

Theorem 14. Let $H_{\nu}(\tau)=\operatorname{Res}\left(Q_{\nu}(\tau \mid s) ; s=2-2 k\right)$, with $\nu \in \mathbb{Z}, \nu \geq 0$, and $0<k<1$. Then $H_{\nu}(\tau) \in C^{0}(k, v)$ and has the following Fourier expansion:

$$
H_{\nu}(\tau)=4 \pi i^{-k} 2^{k} \sum_{n=0}^{\infty} \operatorname{Res}\left(Z_{\nu, n}(w) ; 1-k / 2\right) e^{2 \pi i(n+\kappa) \tau}, \quad \tau \in \mathcal{H} .
$$

Proof. From Lemma 5 and the analysis of the zeta-functions given in the proof of Lemma 13 we have

$$
\begin{aligned}
H_{\nu}(\tau)= & 2 y^{1-k} i^{-k} \frac{(2 \pi)^{2-k}}{\Gamma(1-k)} \sum_{n=0}^{\infty}(n+\kappa)^{1-k} \sigma(4 \pi(n+\kappa) y, 1,1-k) \\
& \times \operatorname{Res}\left(Z_{\nu, n}(s / 2+k / 2) ; 2-2 k\right) e^{2 \pi i(n+\kappa) \tau}, \quad \tau \in \mathcal{H} .
\end{aligned}
$$

Now, this and the fact that $\sigma(4 \pi(n+\kappa) y, 1,1-k)=\Gamma(1-k) /[4 \pi(n+\kappa) y]^{1-k}$ give us the promised Fourier expansion. Notice that $H_{\nu}(\tau)$ is indeed analytic in $\tau$. The modular relation for $H_{\nu}$ is an immediate consequence of (13), Lemma 13 and the principle of analytic continuation.

\section{Decomposition and basis theorems}

Theorem 15. Let $H_{\nu}(\tau)$ be defined as in Theorem 14 , where $\nu \in \mathbb{Z}, \nu \geq$ 0 , and $0<k<1$. Also, let $C_{H}^{0}(\mu, k, v)$ denote the space spanned by $\left\{H_{\nu}\right\}_{\nu=0}^{\mu}$. Then there exists $\mu^{*} \in \mathbb{Z}, \mu^{*} \geq 0$, such that $C_{H}^{0}\left(\mu^{*}, k, v\right)=C^{0}(k, v)$. That is, every cusp form on $\Gamma(1)$ of weight $k$ and $M S v$ is a linear combination of $H_{0}, \ldots, H_{\mu^{*}}$.

Pr o of. We employ a modification of the standard method which shows the completeness of Poincaré series of weight greater than two. Let $f \in$ $C^{0}(k, v)$. We claim that

$$
\left\langle f, H_{\nu}\right\rangle=4 \alpha_{\nu}
$$

where

$$
f(\tau)=\sum_{n=0}^{\infty} \alpha_{n} e^{2 \pi i(n+\kappa) \tau} .
$$

Here we are using the usual Petersson inner product

$$
\left\langle f_{1}, f_{2}\right\rangle=\iint_{\mathcal{R}} f_{1}(\tau) \overline{f_{2}(\tau)} y^{k} \frac{d x d y}{y^{2}}, \quad \tau=x+y i,
$$


where $\mathcal{R}$ is a fundamental region for $\Gamma(1)$. (Note that in general the inner product is valid for $f_{1} \in C\left(\mu_{1}, k, v\right), f_{2} \in C\left(\mu_{2}, k, v\right)$ and $\mu_{1}+\mu_{2}<0$.) In order to prove the claim we consider $\left\langle f, Q_{\nu}\right\rangle$, where $Q_{\nu}$ is given by (12). A calculation, which uses (13) and involves Rankin's unfolding method, gives

$$
\left\langle f, Q_{\nu}\right\rangle=2 \alpha_{\nu} \Gamma(k-1+\bar{s} / 2)[4 \pi(\nu+\kappa)]^{1-k-\bar{s} / 2},
$$

valid for $\operatorname{Re}(s)>2-k$. It follows that

$$
\left\langle f,(s-2+2 k) Q_{\nu}\right\rangle=4 \alpha_{\nu} \Gamma(k+\bar{s} / 2)[4 \pi(\nu+\kappa)]^{1-k-\bar{s} / 2} .
$$

The right-hand side of (15) is analytic, in $\bar{s}$, for $\operatorname{Re}(s)>-2 k$. By Lemma 13, for fixed $\tau \in \mathcal{H}$, the function $(s-2+2 k) Q_{\nu}(\tau \mid s)$ is analytic in $s$ for $\operatorname{Re}(s) \geq$ $2-2 k$. Also, for fixed $s$ with $\operatorname{Re}(s) \geq 2-2 k$, we find that $Q_{\nu}(\tau \mid s)$ vanishes exponentially in $\tau$ as $\operatorname{Im}(\tau) \rightarrow \infty$. These facts, along with the exponential decay of $f$ at $i \infty$, imply that $\left\langle f,(s-2+2 k) Q_{\nu}\right\rangle$ is analytic in $\bar{s}$ for $\operatorname{Re}(s) \geq$ $2-2 k$. So both sides of $(15)$ are analytic in $\operatorname{Re}(s) \geq 2-2 k$. Analytic continuation allows us to set $s=2-2 k$ in (15) and establishes (14).

We now finish our proof through traditional reasoning. By Theorem 14 the space spanned by $\left\{H_{\nu}\right\}_{\nu=0}^{\infty}$ is a subspace of $C^{0}(k, v)$. Our claim shows that the orthogonal complement of this subspace is necessarily trivial. Because $C^{0}(k, v)$ is finite-dimensional there must exist a nonnegative integer $\mu^{*}$ such that $C_{H}^{0}\left(\mu^{*}, k, v\right)=C^{0}(k, v)$.

TheOrem 16. Let $G_{\nu}(\tau)$ be defined as in Lemma 8, and assume that $\nu \in \mathbb{Z}, \nu \geq 0$, and $1<k<2$. Also, let $C_{G}^{0}(\mu, k, v)$ denote the space spanned by $\left\{G_{\nu}\right\}_{\nu=0}^{\mu}$. Then there exists $\mu^{* *} \in \mathbb{Z}, \mu^{* *} \geq 0$, such that $C_{G}^{0}\left(\mu^{* *}, k, v\right)=$ $C^{0}(k, v)$. That is, every cusp form on $\Gamma(1)$ of weight $k$ and $M S v$ is a linear combination of $G_{0}, \ldots, G_{\mu^{* *}}$.

Pro of. The proof of Knopp [6] for $4 / 3<k<2$ carries over to the range $1<k<2$.

TheOREM 17. Let $G_{\nu}(\tau)$ be defined as in Lemma 8, and assume that $\nu \in \mathbb{Z}, \nu<0$, and $1<k<2$. Also, let $W(\mu, k, v), \mu \in \mathbb{Z}^{+}$, denote the space spanned by $\left\{G_{\nu}\right\}_{\nu=-1}^{-\mu}$. Then $I(\mu, k, v)=W(\mu, k, v) \oplus C^{0}(k, v)$. That is, every Niebur modular integral on $\Gamma(1)$ of weight $k$ and $M S v$ can be uniquely written as the sum of a cusp form and a finite linear combination of the functions $G_{\nu}(\tau)$.

P r o o f. We obviously have $W(\mu, k, v) \oplus C^{0}(k, v) \subset I(\mu, k, v)$ and so it remains to prove the reverse inclusion. We shall first show that $\operatorname{dim} I(\mu, k, v) \leq$ $\operatorname{dim} W(\mu, k, v)+\operatorname{dim} C^{0}(k, v)$ for $\mu$ sufficiently large. Recall that $C(\mu, k, v)$ is a subspace of $I(\mu, k, v)$ and consider $I(\mu, k, v) / C(\mu, k, v)$. Now, each Niebur modular integral has a unique cusp form (of complementary weight and conjugate MS) corresponding to it. In fact, there exists a homomorphism from $I(\mu, k, v)$ into $C^{0}(2-k, \bar{v})$ with kernel $C(\mu, k, v)$. A basic homomorphism 
theorem tells us that $\operatorname{dim} I(\mu, k, v) / C(\mu, k, v) \leq \operatorname{dim} C^{0}(2-k, \bar{v})$. Because both $C^{0}(2-k, \bar{v})$ and $C(\mu, k, v)$ are finite-dimensional, so is $I(\mu, k, v)$ and

$$
\operatorname{dim} I(\mu, k, v) \leq \operatorname{dim} C(\mu, k, v)+\operatorname{dim} C^{0}(2-k, \bar{v}) .
$$

We next invoke Petersson's Riemann-Roch Theorem for automorphic forms [13, p. 188]. A double application of this theorem gives

$$
\begin{array}{r}
\operatorname{dim} C(\mu, k, v)-\operatorname{dim} C^{0}(k, v) \\
=\mu+\operatorname{dim} C(-\mu, 2-k, \bar{v})-\operatorname{dim} C^{0}(2-k, \bar{v}) .
\end{array}
$$

From (16) and (17) it follows that

$$
\operatorname{dim} I(\mu, k, v) \leq \mu+\operatorname{dim} C^{0}(k, v)+\operatorname{dim} C(-\mu, 2-k, \bar{v}) .
$$

Now, since $\operatorname{dim} C^{0}(2-k, \bar{v})<\infty$, there exists an $N$ such that

$$
\operatorname{dim} C(-\mu, 2-k, \bar{v})=0 \quad \text { for } \mu \geq N .
$$

Since $\operatorname{dim} W(\mu, k, v)=\mu$ we get

$$
\operatorname{dim} I(\mu, k, v) \leq \operatorname{dim} W(\mu, k, v)+\operatorname{dim} C^{0}(k, v) \quad \text { for } \mu \geq N .
$$

This shows that $I(\mu, k, v)=W(\mu, k, v) \oplus C^{0}(k, v)$ for $\mu \geq N$. We next assume that $\mu<N$ and that $G \in I(\mu, k, v)$. Hence, $G \in I(N, k, v)=$ $W(N, k, v) \oplus C^{0}(k, v)$ and because $G$ has order at $\infty$ greater than or equal to $-\mu+\kappa$ we must have $G \in W(\mu, k, v) \oplus C^{0}(k, v)$. Therefore $I(\mu, k, v) \subset$ $W(\mu, k, v) \oplus C^{0}(k, v)$ and so $I(\mu, k, v)=W(\mu, k, v) \oplus C^{0}(k, v)$ for all $\mu \geq 1$.

11. Special cases. In this section we relate our work to some established results concerning modular forms on $\Gamma(1)$ of small weight. We first focus on cusp forms, which are actually rather rare. Specifically, it is known (see, for example, [8, pp. 349-350]) that $C^{0}(k, v), 0<k<2$, is either 0 -dimensional or is spanned by only one function, $\eta^{2 k}(\tau)$, where

$$
\eta(\tau)=e^{\pi i \tau / 12} \prod_{l=1}^{\infty}\left(1-e^{2 \pi i l \tau}\right), \quad \tau \in \mathcal{H}
$$

is the Dedekind eta-function. The latter happens for only one out of the six possible MS for $\Gamma(1)$ in weight $k$. These facts facilitate the following

TheOREM 18. Let $\eta(\tau)$ be defined as above and let $v_{\eta}$ be the $M S$ associated with $\eta(\tau)$.

(a) For $0<k<1$ and $v \equiv v_{\eta}^{2 k}$ we have

$\eta^{2 k}(\tau)=e^{2 \pi i k \tau / 12}+C_{k} \sum_{n=1}^{\infty} \operatorname{Res}(Z(w ; 0, n, k, v) ; 1-k / 2) e^{2 \pi i(n+k / 12) \tau}, \quad \tau \in \mathcal{H}$.

Here $C_{k}=[\operatorname{Res}(Z(w ; 0,0, k, v) ; 1-k / 2)]^{-1}$ and $Z(w ; 0, n, k, v), n \geq 0$, is Selberg's Kloosterman zeta-function. 
(b) For $1<k<2$ and $v \equiv v_{\eta}^{2 k}$ we have

$$
\eta^{2 k}(\tau)=e^{2 \pi i k \tau / 12}+D_{k} \sum_{n=1}^{\infty} a_{n}(0, k, v) e^{2 \pi i(n+k / 12) \tau}, \quad \tau \in \mathcal{H}
$$

Here $D_{k}=\left[2+a_{0}(0, k, v)\right]^{-1}$ and $a_{n}(0, k, v), n \geq 0$, is as given in Theorem 9 .

Proof. We first prove part (a). It suffices to show that the cusp form $H_{0}=H_{0}\left(\tau ; k, v_{\eta}^{2 k}\right)$ is nontrivial. The given expansion will then follow from Theorem 14. Assume to the contrary that $H_{0}$ vanishes identically and consider the Petersson inner product $\left\langle\eta^{2 k}, H_{0}\right\rangle$. Now, the definition of $\eta(\tau)$ and the inner product formula (14) tell us that $\left\langle\eta^{2 k}, H_{0}\right\rangle=4$. This contradicts our assumption and shows part (a). The proof of part (b) is quite similar and the expansion is from Theorem 9.

COROllaRY 19. Let the Niebur modular integral $G_{\nu}(\tau)=G_{\nu}(\tau ; k, v)$ be defined as before, with $\nu<0$ and $1<k<2$. Also, let $v_{\eta}$ be the $M S$ associated with $\eta(\tau)$. all $\nu$.

(a) If $v \equiv v_{\eta}^{2 k+4 j}, j \in\{0,1,2,3,4\}$, then $G_{\nu}(\tau)$ is a modular form for

(b) If $v \equiv v_{\eta}^{2 k+20}$, then $G_{-1}(\tau)$ is not a modular form.

Proof. This follows from Theorem 11 and Theorem 18(a).

If $\operatorname{dim} C^{0}(k, v)>0$, then in general it is rather difficult to ascertain whether a cusp form which arises from a Poincaré series is nontrivial or is mockingly an "expansion of zero". For small half-integral weights, however, we can establish

TheOREM 20. Assume that $\nu \geq 0$ and let the cusp forms $H_{\nu}(\tau ; k, v)$ and $G_{\nu}(\tau ; k, v)$ be defined as before.

(a) $H_{\nu}\left(\tau ; 1 / 2, v_{\eta}\right)$ is nontrivial if and only if $\nu=m(3 m+1) / 2, m \in \mathbb{Z}$.

(b) $G_{\nu}\left(\tau ; 3 / 2, v_{\eta}^{3}\right)$ is nontrivial if and only if $\nu=m(m+1) / 2, m \in \mathbb{Z}$.

Pr o of. Part (a) follows from (14) and the Fourier expansion

$$
\eta(\tau)=e^{\pi i \tau / 12} \sum_{m=-\infty}^{\infty}(-1)^{m} e^{\pi i m(3 m+1) \tau}, \quad \tau \in \mathcal{H},
$$

and part (b) follows similarly from the expansion

$$
\eta^{3}(\tau)=e^{\pi i \tau / 4} \sum_{m=0}^{\infty}(-1)^{m}(2 m+1) e^{\pi i m(m+1) \tau}, \quad \tau \in \mathcal{H} .
$$

These are essentially the celebrated identities of Euler and Jacobi, respectively (see, for example, [7, pp. 34-39]). 
Corollary 21. Assume now that $\nu<0$ and let the Niebur modular integral $G_{\nu}(\tau ; k, v)$ be defined as before. Then $G_{\nu}\left(\tau ; 3 / 2, v_{\eta}^{23}\right)$ is not a modular form if and only if $\nu=-m(3 m+1) / 2-1, m \in \mathbb{Z}$.

Proof. This is an immediate consequence of the previous theorem and Theorem 11. Note that $v_{\eta}^{23}=v_{\eta}^{-1}$ is the conjugate MS of $v_{\eta}$.

Corollaries 19 and 21 tell us that "almost surely" $G_{\nu}(\tau ; 3 / 2, v)$ is a modular form. The validity of this statement for weight $k, 1<k<2$, depends solely upon whether the cusp form $\eta^{4-2 k}(\tau)$ is lacunary.

Acknowledgements. The author thanks his mentor and friend Marvin Knopp.

\section{References}

[1] D. Goldfeld and P. Sarnak, Sums of Kloosterman sums, Invent. Math. 71 (1983), 243-250.

[2] I. S. Gradshteyn and I. M. Ryzhik, Tables of Integrals, Series, and Products, 5th ed., Academic Press, New York, 1994.

[3] M. Knopp, Fourier series of automorphic forms of nonnegative dimension, Illinois J. Math. 5 (1961), 18-42.

[4] -, Automorphic forms of nonnegative dimension and exponential sums, Michigan Math. J. 7 (1960), 257-287.

[5] - On the Fourier coefficients of small positive powers of $\theta(\tau)$, Invent. Math. 85 (1986), 165-183.

[6] - On the Fourier coefficients of cusp forms having small positive weight, in: Proc. Sympos. Pure Math. 49, Part 2, Amer. Math. Soc., Providence, RI, 1989, 111-127.

[7] —, Modular Functions in Analytic Number Theory, Chelsea, New York, 1993.

[8] J. Lehner, Discontinuous Groups and Automorphic Functions, Amer. Math. Soc., Providence, RI, 1964.

[9] D. Niebur, Automorphic integrals of arbitrary positive dimension and Poincaré series, Doctoral Dissertation, University of Wisconsin, Madison, 1968.

[10] - Construction of automorphic forms and integrals, Trans. Amer. Math. Soc. 191 (1974), 373-385.

[11] H. Petersson, Theorie der automorphen Formen beliebiger reeller Dimension und ihre Darstellung durch eine neue Art Poincaréscher Reihen, Math. Ann. 103 (1930), 369-436.

[12] —, Über die Entwicklungskoeffizienten der automorphen Formen, Acta Math. 58 (1932), 169-215.

[13] - , Automorphe Formen als metrische Invarianten, Math. Nachr. 1 (1948), 158-212.

[14] W. Pribitkin, The Fourier coefficients of modular forms and modular integrals having small positive weight, Doctoral Dissertation, Temple University, Philadelphia, 1995.

[15] H. Rademacher, The Fourier coefficients of the modular invariant $J(\tau)$, Amer. J. Math. 60 (1938), 501-512.

[16] - The Fourier series and the functional equation of the absolute modular invariant $J(\tau)$, ibid. 61 (1939), 237-248.

[17] -, Correction, ibid. 64 (1942), 456. 
[18] H. Rademacher and H. S. Zuckerman, On the Fourier coefficients of certain modular forms of positive dimension, Ann. of Math. 39 (1938), 433-462.

[19] W. Roelcke, Das Eigentwertproblem der automorphen Formen in der hyperbolischen Ebene I, Math. Ann. 167 (1966), 292-337.

[20] A. Selberg, On the estimation of Fourier coefficients of modular forms, in: Theory of Numbers, Proc. Sympos. Pure Math. 8, Amer. Math. Soc., Providence, RI, 1965, $1-15$.

Department of Mathematics and Natural Sciences

Centenary College

Hackettstown, NJ 07840, U.S.A.

E-mail: w_pribitkin@msn.com

Received on 19.8.1997

and in revised form on 21.7.1998 